\title{
Vacuum stability and supersymmetry at high scales with two Higgs doublets
}

\author{
E. Bagnaschi, ${ }^{a}$ F. Brümmer, ${ }^{b}$ W. Buchmüller, ${ }^{a}$ A. Voigt ${ }^{a}$ and G. Weiglein ${ }^{a}$ \\ ${ }^{a}$ Deutsches Elektronen-Synchrotron DESY, \\ Notkestraße 85, D-22607 Hamburg, Germany \\ ${ }^{b}$ Laboratoire Univers et Particules de Montpellier, UMR5299, Université de Montpellier, \\ Place Eugène Bataillon, 34095 Montpellier, France \\ E-mail: emanuele.bagnaschi@desy.de, felix.bruemmer@umontpellier.fr, \\ wilfried.buchmueller@desy.de, alexander.voigt@desy.de, \\ georg.weiglein@desy.de
}

ABSTRACT: We investigate the stability of the electroweak vacuum for two-Higgs-doublet models with a supersymmetric UV completion. The supersymmetry breaking scale is taken to be of the order of the grand unification scale. We first study the case where all superpartners decouple at this scale. We show that contrary to the Standard Model with one Higgs doublet, matching to the supersymmetric UV completion is possible if the lowscale model contains two Higgs doublets. In this case vacuum stability and experimental constraints point towards low values of $\tan \beta \lesssim 2$ and pseudoscalar masses of at least about a $\mathrm{TeV}$. If the higgsino superpartners of the Higgs fields are also kept light, the conclusions are similar and essentially independent of the higgsino mass. Finally, if all gauginos are also given electroweak-scale masses (split supersymmetry with two Higgs doublets), the model cannot be matched to supersymmetry at very high scales when requiring a $125 \mathrm{GeV}$ Higgs. Light neutral and charged higgsinos therefore emerge as a promising signature of a supersymmetric UV completion of the Standard Model at the grand unification scale.

KEYWORDS: Supersymmetry Phenomenology

ArXiv EPrint: 1512.07761 


\section{Contents}

1 Introduction 1

2 THDM models as effective field theories 3

2.1 Preliminary remarks 3

2.2 Conventions for the THDM 4

2.3 Matching to the MSSM at the scale $M_{S}$

2.4 Running to the scale $M_{t} \quad 6$

2.5 Matching at the weak scale $\quad 7$

$\begin{array}{lll}2.6 & \text { Higgs-mass predictions } & 7\end{array}$

$\begin{array}{llr}3 & \text { Results } & \mathbf{8}\end{array}$

3.1 The pure THDM 8

$\begin{array}{ll}3.2 \text { The THDM with higgsinos } & 11\end{array}$

$\begin{array}{lll}3.3 & \text { The THDM with split supersymmetry } & 11\end{array}$

4 Summary and outlook $\quad 13$

$\begin{array}{ll}\text { A Details on the matching at the weak scale } & 14\end{array}$

$\begin{array}{ll}\text { B Vacuum (meta)stability } & 17\end{array}$

\section{Introduction}

The structure of the electroweak and strong interactions seems to point towards an increase of symmetry and to a unification of the fundamental forces as we probe shorter and shorter distances. It is then natural to expect that symmetries larger than the internal and space-time symmetries of the Standard Model of particle physics, including supersymmetry, grand unification and additional space-time dimensions, will play a crucial role for the embedding of the Standard Model into a more fundamental theory. In particular string theory, the leading candidate for a unified theory of all interactions, relies on supersymmetry to guarantee a perturbatively controlled stable vacuum state $[1,2]$. From the point of view of superstring theory, the generic expectation for the scale of supersymmetry breaking is at or close to the string scale, which is of course usually many orders of magnitude larger than the electroweak scale. The Standard Model, possibly supplemented by other light states, would then be the non-supersymmetric effective field theory of a UV completion with spontaneously broken supersymmetry. This UV completion would take effect at a very high energy scale of about $10^{15-17} \mathrm{GeV}$. Example scenarios include universal highscale supersymmetry [3] and split supersymmetry $[4,5]$, which has been realised in string theory [6] and higher-dimensional field theory with flux [7]. 
In the past, the main motivation to consider supersymmetric extensions of the Standard Model used to be the hierarchy problem: electroweak-scale supersymmetry allows to stabilise a large hierarchy between the electroweak scale and a much higher fundamental scale against radiative corrections. However, so far the data shows no sign of supersymmetry. Should no evidence in its favour surface during the second run of the LHC, one may have to conclude that the electroweak scale is not actually protected by supersymmetry, but fixed by some unknown ultraviolet dynamics at a value which presently appears unnatural to us. Our hypothesis for the present paper is that supersymmetry does exist but, since the scale of its breaking is high, that it plays no role in stabilising the electroweak hierarchy.

Admitting a supersymmetric UV completion at high scales is a nontrivial constraint on the low-energy effective theory. For example, it is well known that the Standard Model by itself cannot be matched to its minimal supersymmetric extension (MSSM) above about $10^{11} \mathrm{GeV}[8]$. This is because at higher energies the running Higgs quartic coupling in the Standard Model becomes negative, while the D-term potential in supersymmetry is positive definite. The maximal matching scale is even lower for split supersymmetry, where the electroweak-scale spectrum consists of the Standard Model and the MSSM gauginos and higgsinos [8, 9]. Therefore, to allow for a supersymmetric UV completion at scales of $10^{15-17} \mathrm{GeV}$, more states need to be kept light in the low-energy theory, besides the Standard Model Higgs doublet and possibly gauginos and higgsinos.

Our ability to extrapolate some non-supersymmetric low-energy effective theory to high energies may also be limited by vacuum stability. This, again, is already seen in the Standard Model itself: as a result of the quartic coupling turning negative, the Higgs potential becomes unbounded from below (although the lifetime of the electroweak vacuum has been estimated to be longer than the age of the universe, see ref. [10] and references therein). More generally, demanding a stable or at least sufficiently long-lived vacuum imposes additional constraints on any low-energy theory, even if it can be matched to a supersymmetric UV completion. Although supersymmetry ensures that the potential will be positive definite at the UV completion scale, the RG-improved tree-level potential may still be formally unbounded from below at intermediate energies when expressed in terms of the running couplings. This would signal the presence of additional vacua which are in general deeper than the realistic electroweak vacuum.

A particularly interesting class of models retains both MSSM Higgs doublets as light states at low energies, with or without the light higgsinos and gauginos of split supersymmetry. The matching of the two-Higgs-doublet model (THDM) to the MSSM at high energies has previously been discussed in ref. [11]. Recently, a detailed analysis of the matching for a variety of THDM models as function of the supersymmetry breaking scale has been performed in ref. [12], however, without taking vacuum stability constraints into account. With regards to vacuum stability, the extrapolation of a THDM to high energies near the Planck scale was studied in refs. [13-16], but without imposing constraints from high-energy supersymmetry.

In the present paper we show that several kinds of two-Higgs-doublet models can indeed be matched to GUT-scale or even to string-scale supersymmetry without suffering from vacuum instability. We study three exemplary models using the spectrum gener- 
ator framework FlexibleSUSY [17]: a pure type-II THDM, the THDM with additional electroweak-scale higgsinos (which has the appealing property of gauge coupling unification at $10^{14} \mathrm{GeV}$ ), and the THDM with the full gaugino and higgsino field content of split supersymmetry at the electroweak scale. It turns out that the combined requirements of a supersymmetric UV completion, a stable vacuum, and a $125 \mathrm{GeV}$ Higgs are quite restrictive on the low-energy spectrum. For the pure THDM we find that the parameter region at low $\tan \beta$ and relatively large $M_{A}$, namely $\tan \beta \lesssim 2$ and $M_{A} \gtrsim 1 \mathrm{TeV}$, is in agreement with all these constraints as well as with the experimental bounds from the measurement of $\mathrm{BR}(b \rightarrow s \gamma)[18]$ and the limits from the searches for additional Higgs bosons, in particular in the channel $H, A \rightarrow \tau \tau$ [19-21]. The conclusions are similar but somewhat more restrictive for the THDM with light Higgsinos. For the THDM with split supersymmetry, on the other hand, we find that the model cannot be extrapolated to the scale of Grand Unification because the predicted mass of the Standard Model-like Higgs boson is always too large in the parameter regions allowed by the other constraints.

\section{THDM models as effective field theories}

\subsection{Preliminary remarks}

The standard procedure for treating theories with several hierarchically separated scales is to "run and match" the effective field theory parameters. That is, the theory is regularised and renormalised using the $\overline{\mathrm{MS}}$ scheme (or one of its cousins such as $\overline{\mathrm{DR}}$ ), the parameters are evolved according to their $n$-loop renormalisation group equations in between the thresholds, and at each threshold crossing the heavy states are decoupled by hand. The parameters of the resulting effective theory are matched to those of the full theory with $(n-1)$-loop precision. If the masses of two heavy states are comparable to each other, they should be decoupled simultaneously and their mass difference accounted for by an appropriate threshold correction at leading-log order. If on the other hand the masses of two heavy states are widely separated, then they define two distinct thresholds between which the logarithms should be resummed, using the renormalisation group equations of an intermediate effective theory.

For the present study we will always use precisely one effective field theory between the supersymmetry breaking scale $M_{S}=10^{15-17} \mathrm{GeV}$ and the electroweak scale. While intermediate thresholds certainly offer interesting possibilities to generalise our work, here we will always assume that one set of particles decouples close to $M_{S}$ and that the remaining states will obtain masses at most of the order of a TeV. These "light" states will always include the Standard Model particles and a second Higgs doublet; we will furthermore investigate the cases where they also include a pair of higgsinos, or a pair of higgsinos and all MSSM gauginos.

In particular, we take all the eigenvalues of the Higgs mass matrix to be comparable to each other, and therefore the running parameters of the THDM must be matched directly to the measured pole masses of the Standard Model particles. Thus, our study differs from the often considered case where the mass scale $M_{A}$ of the non-standard Higgs bosons is much higher than the electroweak scale. In this case the appropriate procedure would be 
to decouple the non-standard Higgs bosons at the high scale $M_{A}$, to match the THDM to the Standard Model at $M_{A}$, and then to evolve the Standard Model running parameters down to the electroweak scale.

Imposing that all Higgs bosons acquire masses $\lesssim \mathrm{TeV}$ is a strong assumption, which as discussed above is technically unnatural since as for the discovered Higgs boson at $125 \mathrm{GeV}$ also the masses of further relatively light Higgs bosons should be affected by highscale physics. The Higgs mass parameters of the low-energy theory are determined by the matching conditions to the unknown supersymmetric theory at $M_{S} \sim 10^{15-17}$, and are generically expected to be of the order of $M_{S}$ itself. Here we postulate that the various contributions to the Higgs mass matrix cancel each other to a very high degree of precision, such that all of its entries are of the order of at most a TeV. We refrain from speculating about the reasons - in our approach we assume that the hierarchy problem is solved by the UV theory by some means unknown to us. It has been argued that the electroweak scale might need to be low for anthropic reasons, and that this would predict precisely one light scalar doublet. We do not subscribe to these arguments; it seems to us that they rest on rather frail assumptions, and that even if anthropics should indeed be related to the electroweak hierarchy, this would not necessarily preclude a (presently unknown) anthropic argument for a second light Higgs doublet.

\subsection{Conventions for the THDM}

We use the following conventions for parameterising the scalar potential of the THDM as

$$
\begin{aligned}
V= & m_{1}^{2} H_{1}^{\dagger} H_{1}+m_{2}^{2} H_{2}^{\dagger} H_{2}-\left(m_{12}^{2} H_{1}^{\dagger} H_{2}+\text { h.c. }\right)+V_{4}, \\
V_{4}= & \frac{\lambda_{1}}{2}\left(H_{1}^{\dagger} H_{1}\right)^{2}+\frac{\lambda_{2}}{2}\left(H_{2}^{\dagger} H_{2}\right)^{2}+\lambda_{3}\left(H_{1}^{\dagger} H_{1}\right)\left(H_{2}^{\dagger} H_{2}\right)+\lambda_{4}\left|H_{1}^{\dagger} H_{2}\right|^{2} \\
& +\left(\frac{\lambda_{5}}{2}\left(H_{1}^{\dagger} H_{2}\right)^{2}+\lambda_{6}\left(H_{1}^{\dagger} H_{2}\right)\left(H_{1}^{\dagger} H_{1}\right)+\lambda_{7}\left(H_{1}^{\dagger} H_{2}\right)\left(H_{2}^{\dagger} H_{2}\right)+\text { h.c. }\right) .
\end{aligned}
$$

For each Yukawa term allowed in the Standard Model, the general THDM contains two such terms, one involving $H_{1}$ and the other involving $H_{2}$. Moreover, if there are light gauginos $\left(\tilde{B}, \tilde{W}^{i}, \tilde{G}^{a}\right)$ and higgsinos $\left(\tilde{h}_{d}, \tilde{h}_{u}\right)$ in the spectrum, they are coupled to the Higgs doublets with the Yukawa terms

$$
\begin{aligned}
-\mathcal{L}_{\text {Yuk }}= & \frac{\tilde{g}_{d}}{\sqrt{2}} H_{1} \tilde{W} \tilde{h}_{d}+\frac{\tilde{g}_{d}^{\prime}}{\sqrt{2}} H_{1} \tilde{B} \tilde{h}_{d}+\frac{\tilde{g}_{u}}{\sqrt{2}} H_{2}^{\dagger} \tilde{W} \tilde{h}_{u}+\frac{\tilde{g}_{u}^{\prime}}{\sqrt{2}} H_{2}^{\dagger} \tilde{B} \tilde{h}_{u} \\
& +\frac{\tilde{\gamma}_{d}}{\sqrt{2}} H_{2} \tilde{W} \tilde{h}_{d}+\frac{\tilde{\gamma}_{d}^{\prime}}{\sqrt{2}} H_{2} \tilde{B} \tilde{h}_{d}+\frac{\tilde{\gamma}_{u}}{\sqrt{2}} H_{1}^{\dagger} \tilde{W} \tilde{h}_{u}+\frac{\tilde{\gamma}_{u}^{\prime}}{\sqrt{2}} H_{1}^{\dagger} \tilde{B} \tilde{h}_{u} \\
& + \text { h.c.. }
\end{aligned}
$$

The gauge symmetries of the general THDM with higgsinos and gauginos further allow for Yukawa couplings between the higgsinos, right-handed leptons and Higgs bosons.

If all the couplings allowed by gauge symmetry were actually present (and sizeable) in the THDM, this would lead to phenomenologically unacceptable rates of flavour changing neutral currents. However, matching to supersymmetry leads to strong restrictions on the parameter space as we will now describe in detail. 


\subsection{Matching to the MSSM at the scale $M_{S}$}

We identify $H_{1}=-i \sigma^{2} H_{d}^{*}$ and $H_{2}=H_{u}$ at the scale $M_{S}$, where $H_{u}$ and $H_{d}$ are the Higgs doublets of the minimal supersymmetric Standard Model. Tree-level matching at the scale $M_{S}$ gives

$$
\begin{aligned}
& \lambda_{1}=\frac{1}{4}\left(g^{2}+g^{\prime 2}\right), \\
& \lambda_{2}=\frac{1}{4}\left(g^{2}+g^{\prime 2}\right), \\
& \lambda_{3}=\frac{1}{4}\left(g^{2}-g^{\prime 2}\right), \\
& \lambda_{4}=-\frac{1}{2} g^{2}, \\
& \lambda_{5}=\lambda_{6}=\lambda_{7}=0 .
\end{aligned}
$$

Here $g \equiv g_{2}$ and $g^{\prime} \equiv \sqrt{\frac{3}{5}} g_{1}$.

The one-loop threshold corrections to these couplings are e.g. listed in ref. [11]. The exact superpartner spectrum at $M_{S}$ is of course unknown, but we use the GUT model of ref. [7] as a guidance. It predicts that the squark and slepton soft masses are degenerate to leading order at the matching scale $M_{S}$, and that all other soft parameters are generated at subleading order. In this case the squark and slepton threshold corrections are suppressed not only by a loop factor but also by the small ratios $A / M_{S}, \mu / M_{S}$ and by the near-degeneracy of the squarks and sleptons, and their impact on our results is correspondingly reduced.

In the following we set these threshold corrections to zero for definiteness, with the understanding that this is a source of model dependence. To account for the neglected effects, we will assume a conservative $3 \mathrm{GeV}$ uncertainty on $m_{h}$ in our analysis.

Following the same line of reasoning, we also neglect the higgsino threshold corrections to eqs. (2.3) in the pure THDM case, and the electroweak gaugino threshold corrections in the case of both the pure THDM and the THDM with light higgsinos.

Note that the tree level matching conditions eqs. (2.3) are not specific to the UV completion being the MSSM, but apply in any model in which the quartic scalar potential emerges from the $D$-term potential of an $N=1$ supersymmetric $\mathrm{SU}(2) \times \mathrm{U}(1)$ theory.

Since we are setting $\lambda_{5}=\lambda_{6}=\lambda_{7}=0$ in our analysis, and since the Yukawa terms $H_{d}^{\dagger} \bar{u}_{R} q_{L}+H_{u}^{\dagger} \bar{d}_{R} q_{L}+H_{u}^{\dagger} \bar{e}_{R} \ell_{L}+$ h.c. are also absent at the matching scale (up to small threshold corrections which we neglect), our model becomes an effective type-II THDM.

If there are winos or binos in the spectrum, the matching conditions for their Yukawa couplings at the scale $M_{S}$ read at the tree-level

$$
\begin{array}{r}
\tilde{g}_{u}=g, \\
\tilde{g}_{d}=g, \\
\tilde{g}_{u}^{\prime}=g^{\prime}, \\
\tilde{g}_{d}^{\prime}=g^{\prime}, \\
\tilde{\gamma}_{u}=\tilde{\gamma}_{d}=\tilde{\gamma}_{u}^{\prime}=\tilde{\gamma}_{d}^{\prime}=0 .
\end{array}
$$


We will again neglect possible effects from small threshold corrections. We also assume that there is some conserved quantum number (such as $R$-parity or $B-L$ ) distinguishing the higgsino from the lepton doublets, such that there are no Yukawa couplings between the Higgs, the higgsino and the right-handed leptons.

\subsection{Running to the scale $M_{t}$}

The $\lambda_{i}$ evolve from $M_{S}$ down to the electroweak scale according to their renormalisation group equations. Note that $\lambda_{5,6,7}, \tilde{\gamma}_{u, d}, \tilde{\gamma}_{u, d}^{\prime}$, as well as the "wrong Higgs" quark and lepton Yukawa couplings, are protected by the symmetries of the effective theory and therefore will not be generated during the running if they are zero at the matching scale, which we assume is the case. We therefore work with all these couplings set to zero henceforth.

To obtain a scalar potential that is bounded from below, a set of sufficient conditions on the running scalar couplings is ref. [23]

$$
\begin{aligned}
\lambda_{1} & >0, \\
\lambda_{2} & >0, \\
\lambda_{3}+\left(\lambda_{1} \lambda_{2}\right)^{1 / 2} & >0, \\
\lambda_{3}+\lambda_{4}+\left(\lambda_{1} \lambda_{2}\right)^{1 / 2} & >0 .
\end{aligned}
$$

Numerically it will turn out that the first three conditions are always satisfied as a consequence of the supersymmetric matching conditions, while the fourth one eq. (2.8) may be violated at intermediate scales.

The stability conditions can be relaxed if one allows for additional vacua besides the electroweak one, and merely imposes that the lifetime of the electroweak vacuum be $\gtrsim 10^{10}$ years. In that case, assuming that the conditions (2.5)-(2.7) are satisfied, the condition (2.8) is replaced by an inequality which should hold at all renormalisation scales $\mu_{r}$,

$$
\lambda\left(\mu_{r}\right) \gtrsim-\frac{2.82}{41.1+\log _{10} \frac{\mu_{r}}{\mathrm{GeV}}} \equiv \lambda_{\text {meta }},
$$

where

$$
\lambda=\frac{4\left(\lambda_{1} \lambda_{2}\right)^{1 / 2}\left(\lambda_{3}+\lambda_{4}+\left(\lambda_{1} \lambda_{2}\right)^{1 / 2}\right)}{\lambda_{1}+\lambda_{2}+2\left(\lambda_{1} \lambda_{2}\right)^{1 / 2}} .
$$

A derivation of eq. (2.9) is given in appendix B.

In order to numerically study the running of the parameters in the presence of the boundary and vacuum stability conditions, we use the spectrum generator framework FlexibleSUSY 1.2.1 [17] in combination with SARAH 4.6.0 [24-26]. ${ }^{1}$ The latter is used to compute the 2-loop renormalisation group equation for the effective field theories. As a preliminary safety-check, we have compared the expressions obtained from SARAH with the ones provided by PYR@TE [27, 28], finding complete agreement.

FlexibleSUSY makes use of 2-loop renormalisation group equations and provides an automatic matching of the THDM to input parameters at the electroweak scale (we perform the matching at the scale $M_{t}$ ), as described in the following as well as in more detail in appendix A.

\footnotetext{
${ }^{1}$ The SARAH version we use contains an additional bug-fix, which corrects the $\overline{\mathrm{MS}}-\overline{\mathrm{DR}}$ conversion terms in the left- and right-handed one-loop fermion self-energies.
} 


\subsection{Matching at the weak scale}

By integrating the 2-loop renormalisation group equations we obtain the running parameters of the THDM (potentially including higgsinos and gauginos) at the scale $M_{t}$, where we match the THDM to experimentally known input parameters. The matching is performed by calculating the $\overline{\mathrm{MS}}$ gauge and Yukawa couplings as well as the VEVs of the THDM from known input parameters at the 1- and leading 2-loop level. In particular, at the tree level, the well-known THDM relations

$$
\begin{aligned}
m_{12}^{2} & =m_{A}^{2} \sin \beta \cos \beta, \\
m_{1}^{2} & =m_{12}^{2} \tan \beta-v^{2}\left(\lambda_{1} \cos ^{2} \beta+\left(\lambda_{3}+\lambda_{4}\right) \sin ^{2} \beta\right), \\
m_{2}^{2} & =m_{12}^{2} \cot \beta-v^{2}\left(\lambda_{2} \sin ^{2} \beta+\left(\lambda_{3}+\lambda_{4}\right) \cos ^{2} \beta\right),
\end{aligned}
$$

allow us to express the entire scalar potential in terms of $v=\sqrt{v_{u}^{2}+v_{d}^{2}}$, the quartic couplings, the pseudoscalar $\overline{\mathrm{MS}}$ Higgs mass $m_{A}$ and

$$
\tan \beta \equiv \frac{v_{2}}{v_{1}} .
$$

More details on the matching procedure at the loop level are given in appendix A.

We note that our models have the appealing feature that there are very few parameters left in the low-energy theory. Since the quartic couplings are essentially determined by the gauge couplings via the supersymmetric boundary conditions, the only free parameter which directly affects them is the matching scale $M_{S}$. Setting $v \approx 174 \mathrm{GeV}$ implies that, in the pure THDM, the Higgs mass spectrum is completely determined by the parameters $M_{S}, m_{A}$ and $\tan \beta$, one of which can (in principle) be fixed by requiring $M_{h}=125 \mathrm{GeV}$. Moreover, requiring vacuum stability forces us into the region of rather low $\tan \beta$, and the sensitivity of the low-energy spectrum to $M_{S}$ is very mild. This allows us, in principle, to predict a sharp correlation between $\tan \beta$ and $m_{A}$. In practice, however, the theory uncertainty on the calculation of the lightest Higgs mass is still so large that there is still room for significant variation, as we will detail in the next section.

\subsection{Higgs-mass predictions}

In the THDM with higgsinos, the Higgs masses receive loop corrections from charginos and neutralinos and hence depend on the higgsino mass parameter $\mu$. This leads to correlations between the Higgs and neutralino and chargino masses which are in principle testable at colliders. In the THDM with higgsinos and gauginos, the Higgs masses depend on all the chargino and neutralino masses, and may in addition be affected by two-loop corrections from the gluino. This will also become evident in the next section.

We calculate the CP-even Higgs pole masses by numerically finding the two eigenvalues $M_{h, H}^{2}$ of the one-loop-corrected mass matrix

$$
\underline{M}_{h, 1 \mathrm{~L}}^{2}=\underline{M}_{h}^{2}-\operatorname{Re} \Sigma_{h}\left(p^{2}=M_{h, H}^{2}, \mu_{r}=M_{t}\right) .
$$

Here, $\underline{M}_{h}^{2}$ denotes the CP-even Higgs mass matrix expressed in terms of the $\overline{\mathrm{MS}}$ parameters at the scale $\mu_{r}=M_{t}$ and $\Sigma_{h}\left(p^{2}, \mu_{r}\right)$ is the $\overline{\mathrm{MS}}$ renormalised CP-even Higgs one-loop selfenergy matrix, where the Higgs fields at the external legs are taken to be the Higgs gauge 
eigenstates. Since the Higgs self-energy has to be evaluated at the momenta $p^{2}=M_{h, H}^{2}$, where $M_{h, H}^{2}$ are the eigenvalues of $\underline{M}_{h, 1 \mathrm{~L}}^{2}$, eq. (2.13) is solved iteratively.

\section{Results}

\subsection{The pure THDM}

The low-energy parameter space is strongly constrained by vacuum (meta)stability, by requiring the lightest Higgs boson mass to be $125 \mathrm{GeV}$, and by the experimental bounds from the measurement of $\mathrm{BR}(b \rightarrow s \gamma)$ [18] and the limits from the LHC searches for $H, A \rightarrow \tau \tau$ [19-21]. In the top row of figure 1 we show contours of the lightest Higgs mass as a function of $M_{A}$ and $\tan \beta$ for a SUSY breaking scale $M_{S}=2 \cdot 10^{14} \mathrm{GeV}$. The vacuum is absolutely stable only in the white unshaded region at low $\tan \beta$. It is metastable in the bulk of the parameter space (orange regions), and unstable in the red region of intermediate $\tan \beta$.

We remark that including high-scale one-loop threshold corrections from heavy higgsinos, which we have neglected in generating these plots, can have a significant impact on the large $\tan \beta$ region. For example, choosing $\mu=0.1 M_{S}$ somewhat lowers the upper boundary of the unstable region and opens up a new stable region around $\tan \beta=30$. However, the constraint $M_{h}=125 \mathrm{GeV}$ enforces $M_{A} \lesssim 200 \mathrm{GeV}$ at large $\tan \beta$, and this parameter region is excluded by the constraint on the charged Higgs boson mass in a THDM from the measurement of $\mathrm{BR}(b \rightarrow s \gamma)$ (since the charged Higgs is similarly light as the pseudoscalar) and by the limits from the LHC searches for $H, A \rightarrow \tau \tau$. Thus, including or neglecting these threshold corrections only affects a parameter region which is phenomenologically disfavoured anyway.

Note that absolute vacuum stability forces one into the low $\tan \beta$ region, $\tan \beta \lesssim 1.8$, with pseudoscalar Higgs masses exceeding a TeV for $M_{h}=125 \mathrm{GeV}$ and the central value of $M_{t}$. By contrast, when allowing for the vacuum to be metastable, the most severe constraint on $M_{A}$ comes from the measurement of $\mathrm{BR}(b \rightarrow s \gamma)$, which together with the requirement that $M_{h}$ should be close to $125 \mathrm{GeV}$ still points to somewhat small tan $\beta$ values, $\tan \beta \lesssim 5$.

For comparison, we also show the case of a higher SUSY breaking scale $M_{S}=2$. $10^{17} \mathrm{GeV}$ in the bottom row of figure 1 . This scale, an order of magnitude below $M_{\text {Planck }}$, is about the highest for which the matching to a weakly coupled four-dimensional supersymmetric field theory can be justified. While the qualitative behaviour in the plane is the same as for the lower SUSY breaking scale case, we observe that a large part of the formerly metastable region is now unstable. Concerning the higgsino one-loop threshold corrections, similar remarks as above apply. ${ }^{2}$

In order to understand why the THDM allows a matching to the supersymmetric standard model at very high scales one has to study the renormalisation group flow of the quartic couplings. This is shown in figure 2 for $M_{S}=2 \cdot 10^{14} \mathrm{GeV}$ for two values of $\tan \beta$.

\footnotetext{
${ }^{2}$ Note that for part of the parameter space considered in ref. [12], $m_{A}=200 \mathrm{GeV}$ and $M_{S}$ of the order of the grand unification scale, the electroweak vacuum is either metastable or unstable.
} 

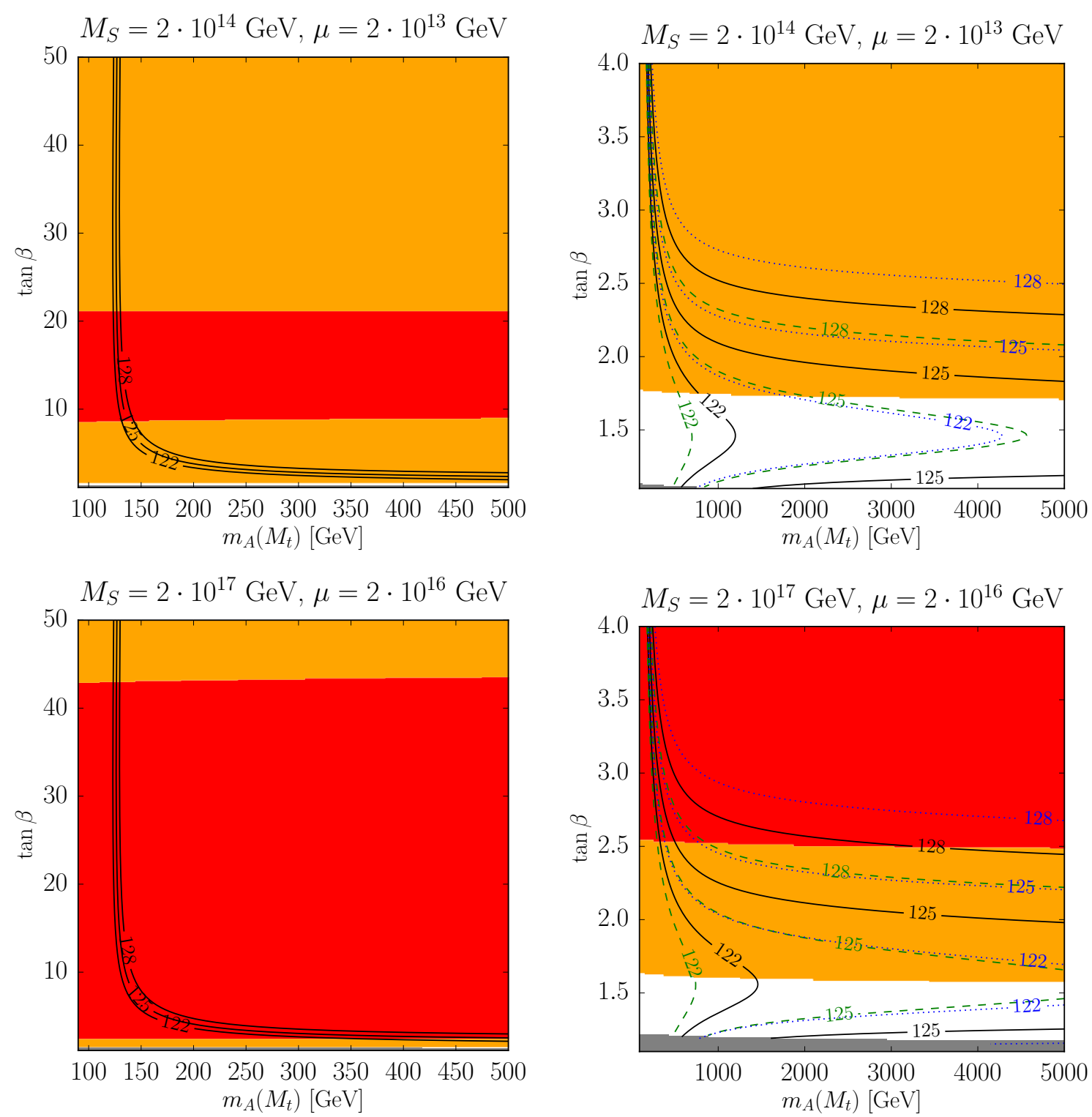

\begin{tabular}{llllll}
- & $M_{t}=173.34 \mathrm{GeV} \quad \cdots$ & $M_{t}=174.1 \mathrm{GeV}$ & $\cdots \cdots \cdots \cdots$ & $M_{t}=172.58 \mathrm{GeV}$ \\
\hline
\end{tabular}

Figure 1. Contours of the lightest Higgs mass $M_{h}$ in the $m_{A}\left(M_{t}\right)-\tan \beta$ plane in the pure THDM for $M_{S}=2 \cdot 10^{14} \mathrm{GeV}$ (top row) and $M_{S}=2 \cdot 10^{17} \mathrm{GeV}$ (bottom row). The Higgs mass prediction is computed for $M_{t}=173.34 \pm 0.76 \mathrm{GeV}$ (solid black, dashed green and dotted blue). Left: full range of $\tan \beta$, low $m_{A}\left(M_{t}\right)$; right: region of low $\tan \beta$, large $m_{A}\left(M_{t}\right)$. Unshaded regions are allowed by vacuum stability. In the orange region, the electroweak vacuum is unstable but its lifetime is larger than the age of the universe. Red regions are excluded by vacuum stability. Grey regions are uncalculable because perturbative control is lost. 

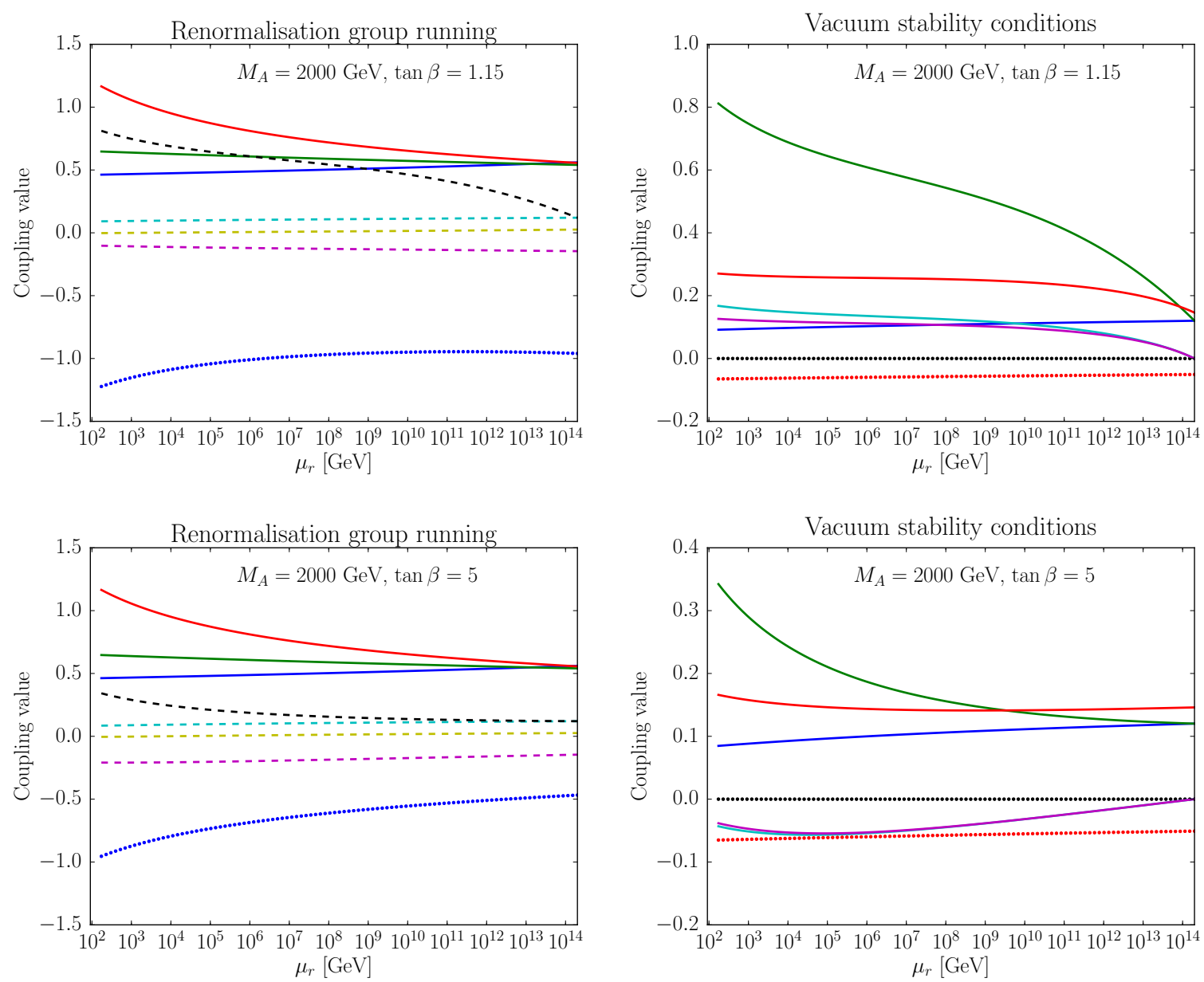

\begin{tabular}{|llllllll|}
\hline-- & $\lambda_{1}$ & $\cdots$ & $\lambda_{3}$ & - & $g_{1}$ & - & $g_{3}$ \\
-- & $\lambda_{2}$ & $\cdots$ & $\lambda_{4}$ & - & $g_{2}$ & $\ldots$ & $y_{t}$ \\
\hline
\end{tabular}

$\begin{array}{llll}- & \lambda_{1} & - & \lambda_{3}+\lambda_{4}+\left(\lambda_{1} \lambda_{2}\right)^{1 / 2} \\ - & \lambda_{2} & - & \lambda \\ -\quad \lambda_{3}+\left(\lambda_{1} \lambda_{2}\right)^{1 / 2} & \ldots & \lambda_{\text {meta }}\end{array}$

Figure 2. Renormalisation group running of dimensionless parameters (left column) and the vacuum stability conditions (right column), in the THDM for $M_{S}=2 \cdot 10^{14} \mathrm{GeV}$, for two different points characterised by a stable electroweak vacuum (top row) and metastable behaviour (bottom row). $\mu_{r}$ denotes the renormalisation scale. $\lambda$ and $\lambda_{\text {meta }}$ are defined in eqs. (2.9) and (2.10).

For small values of $\tan \beta$ the absolute value of the top-quark Yukawa coupling is large in the IR. This drives the coupling $\lambda_{2}$ also to large values in the IR. In the UV, at $M_{S}$, all quartic couplings are determined by the gauge couplings, which approximately unify in the THDM. Due to the boundary conditions the coupling $\lambda_{4}$ is negative at $M_{S}$. Hence the condition $\lambda_{3}+\lambda_{4}+\sqrt{\lambda_{1} \lambda_{2}}>0$ is the most stringent stability constraint. As figure 2 shows, for $\tan \beta=1.15$ the coupling $\lambda_{2}$ is sufficiently large such that $\sqrt{\lambda_{1} \lambda_{2}}$ can compensate the negative $\lambda_{4}$. For $\tan \beta=5$ this is no longer the case, and only the weaker metastability condition is satisfied. 


\subsection{The THDM with higgsinos}

In the case that the gauginos, squarks and sleptons are decoupled at the scale $M_{S}$, while the Higgs bosons of the THDM and their superpartners have masses at the electroweak scale, the low-energy mass spectrum depends on the additional parameter $\mu$. Figure 3 shows the results for $\mu=200 \mathrm{GeV}$; the picture is qualitatively very similar for $\mu=2000 \mathrm{GeV}$. Already at $M_{S}=2 \cdot 10^{14} \mathrm{GeV}$ a wide range of $\tan \beta$ values is now excluded because the vacuum is unstable. For a metastable vacuum the requirement that $M_{h}$ should be close to $125 \mathrm{GeV}$ favours somewhat higher $M_{A}$ values than for the pure THDM, in accordance with the constraint from the measurement of $\operatorname{BR}(b \rightarrow s \gamma)$. An absolutely stable region remains at small values of $\tan \beta$, favouring somewhat higher $M_{A}$ values than in the pure THDM case. For a higher SUSY breaking scale $M_{S}=2 \cdot 10^{17} \mathrm{GeV}$ the parameter space is even more constrained.

It is important to notice that the existence of a stable region at small $\tan \beta$ imposes no constraints on the parameter $\mu$. Hence, a scenario where at the weak scale the particle content of the Standard Model is supplemented by the Higgs bosons of a second doublet at about a $\mathrm{TeV}$ and light neutral and charged higgsinos is fully compatible with the matching to a supersymmetric UV completion at the grand unification scale. A discovery of light higgsinos at the LHC could therefore be interpreted as a possible hint for a supersymmetric UV completion at the grand unification scale.

\subsection{The THDM with split supersymmetry}

When retaining the full gaugino spectrum of the MSSM as well as its complete Higgs sector as the light degrees of freedom, this particle content has the appealing feature that the gauge couplings approximately unify at the scale $M_{\mathrm{GUT}}=2 \cdot 10^{16} \mathrm{GeV}$. The bestmotivated choice for the matching scale in this case is therefore $M_{S}=M_{\mathrm{GUT}}$.

The low-energy spectrum now depends on the gaugino masses $M_{1,2,3}$ as well as on $\mu$. For simplicity we choose a common low-scale value $M_{1}=M_{2}=\mu$ for the electroweak superpartner masses, while keeping $M_{3}=2000 \mathrm{GeV}$ to avoid experimental limits from LHC Run 1. (Alternatively we could have imposed gaugino mass unification at $M_{\mathrm{GUT}}$, which leads to very similar results for a low-scale value of $M_{2}$ equal to $\mu$.) The Higgs sector is affected by the gluino only through two-loop effects, and therefore is not very sensitive to the precise value of $M_{3}$, given that the squarks are decoupled. We can therefore assume that the gluino is sufficiently heavy to have escaped detection at the LHC so far.

We find that in the case of light gauginos the vacuum stability conditions are always satisfied and therefore imply no constraint on $\tan \beta$. As shown in figure 4 , however, a Higgs mass consistent with observation can only be obtained for small values of $M_{A}$ which are essentially excluded by the constraint from the measurement of $\operatorname{BR}(b \rightarrow s \gamma)$ in this scenario. Hence, the extrapolation of the THDM with light higgsinos and gauginos up to the grand unification scale is not compatible with the measured value of the Higgs boson mass. 

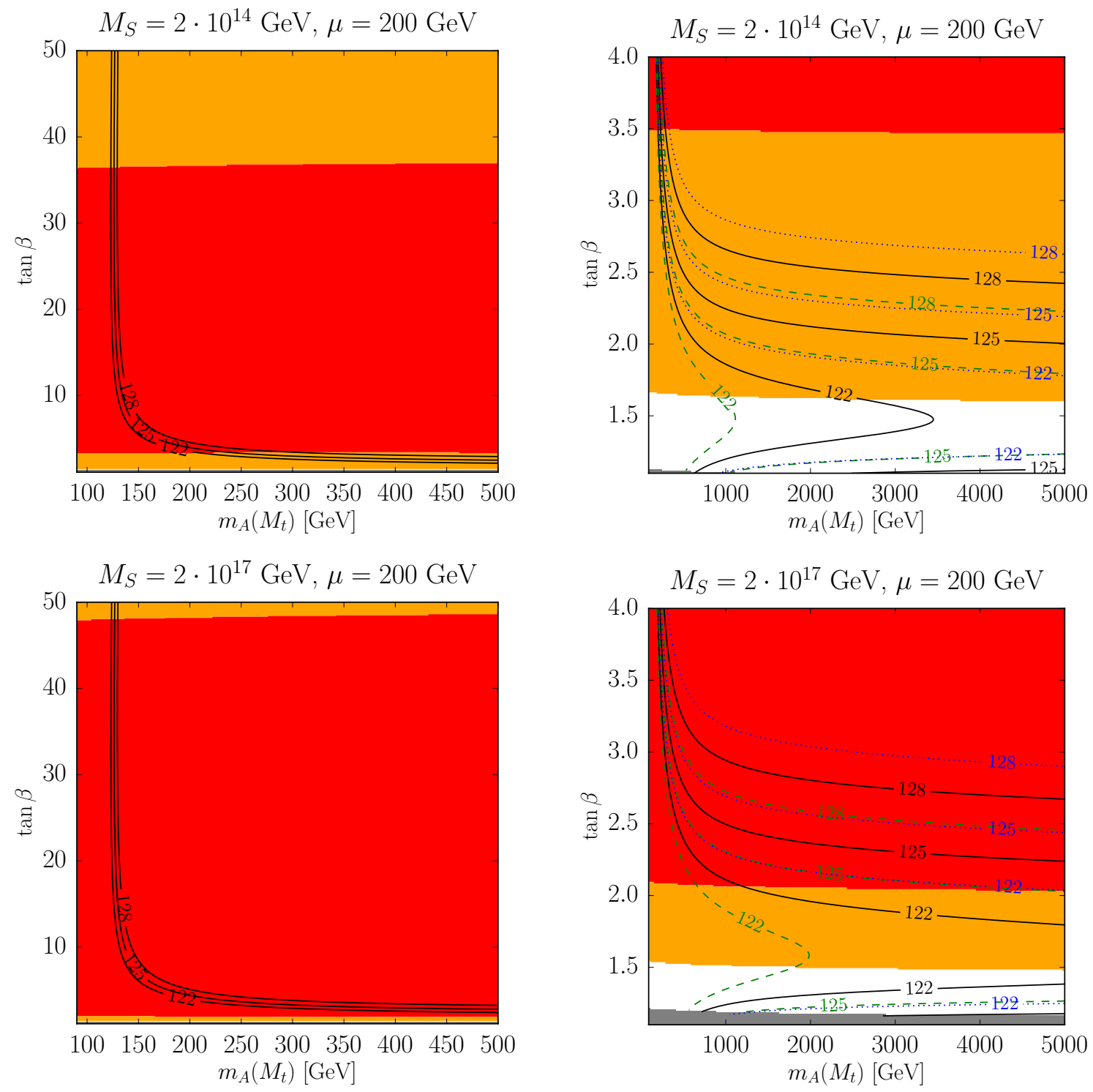

$$
\begin{array}{llllll}
- & M_{t}=173.34 \mathrm{GeV} & \cdots & M_{t}=174.1 \mathrm{GeV} & \cdots \cdots \cdots \cdots & M_{t}=172.58 \mathrm{GeV} \\
\hline
\end{array}
$$

Figure 3. Contours of the lightest Higgs mass $M_{h}$ in the $m_{A}\left(M_{t}\right)-\tan \beta$ plane for the case where the spectrum at the electroweak scale consists of the THDM with higgsinos, with $\mu=200 \mathrm{GeV}$, for $M_{S}=2 \cdot 10^{14} \mathrm{GeV}$ (top row) and $M_{S}=2 \cdot 10^{17} \mathrm{GeV}$ (bottom row). The Higgs mass prediction is computed for $M_{t}=173.34 \pm 0.76 \mathrm{GeV}$ (solid black, dashed green and dotted blue). Left: full range of $\tan \beta$, low $m_{A}\left(M_{t}\right)$; right: region of low $\tan \beta$, large $m_{A}\left(M_{t}\right)$. Unshaded regions are allowed by vacuum stability. In the orange region, the electroweak vacuum is unstable but its lifetime is larger than the age of the universe. Red regions are excluded by vacuum stability. Grey regions are uncalculable because perturbative control is lost. 

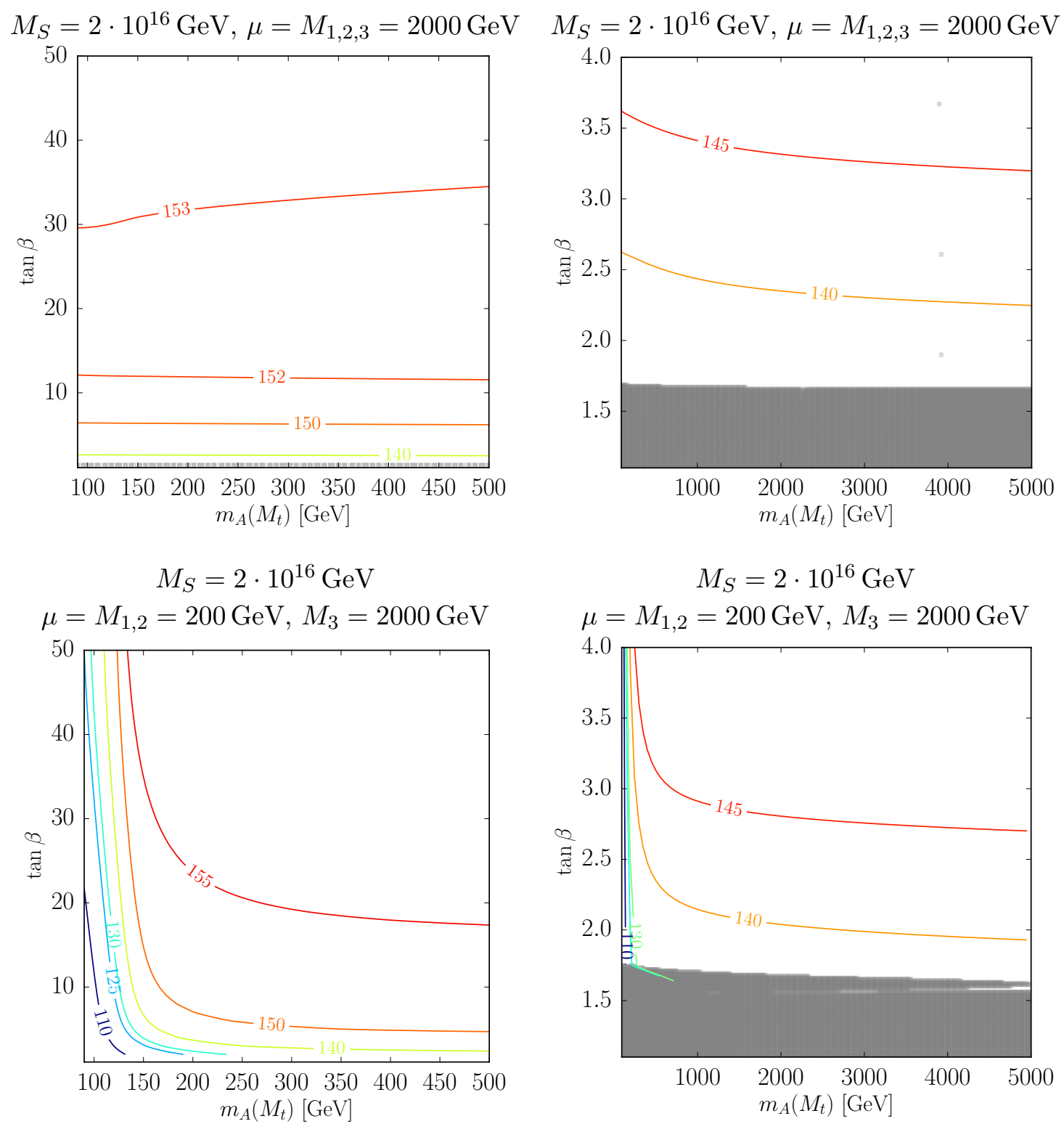

Figure 4. Contours of the lightest Higgs mass $M_{h}$ in the $m_{A}\left(M_{t}\right)-\tan \beta$ plane for the case where the spectrum at the electroweak scale consists of the THDM with gauginos and higgsinos (splitsupersymmetry) for $M_{S}=2 \cdot 10^{16} \mathrm{GeV}$, with $\mu=2000 \mathrm{GeV}$ (top row) and $\mu=200 \mathrm{GeV}$ (bottom row). Left: full range of $\tan \beta$, low $M_{A}$; right: region of low $\tan \beta$, large $M_{A}$. Unshaded white regions are allowed by vacuum stability. Grey regions are uncalculable because perturbative control is lost.

\section{Summary and outlook}

We have studied the matching of the Standard Model, supplemented by a second Higgs doublet, with or without additional higgsinos and gauginos, to the supersymmetric standard model at high scales close to the GUT scale. A supersymmetric ultraviolet completion of the Standard Model is strongly motivated by unified theories, in particular string theory. 
The extrapolation of the Standard Model to high scales is severely constrained by the necessary requirement of stability or metastability of the electroweak vacuum. In the Standard Model a matching to its supersymmetric extension at the GUT scale is not possible for the measured mass of the Higgs boson. On the contrary, as we have shown, a matching consistent with vacuum stability is possible for two-Higgs-doublet models. For small values of $\tan \beta$ the large top-quark Yukawa coupling drives one of the quartic Higgs couplings to large values in the IR. As a consequence, all vacuum stability conditions can be satisfied.

The matching of the pure THDM to its supersymmetric extension at high scales implies a lower bound on the additional Higgs boson masses of about a TeV. This bound shows a significant sensitivity on the remaining theoretical uncertainties induced by the experimental error of the mass of the top quark and from unknown higher-order corrections. In case of light higgsinos the lower bound is slightly more stringent than for the case of the pure THDM. Because of this preference for low values of $\tan \beta$ and relatively high values of $M_{A}$, the discovery of additional Higgs bosons at the LHC appears challenging in this scenario. In principle, smaller pseudoscalar masses can be consistent with a metastable electroweak vacuum. But these values of $M_{A}$ are already essentially excluded by the constraints from rare processes. Finally, in the case of both higgsinos and gauginos at the $\mathrm{TeV}$ scale the vacuum stability conditions are always fulfilled, but a Higgs mass of $125 \mathrm{GeV}$ implies values of $M_{A}$ that are incompatible with low energy measurements.

It is remarkable that the extrapolation of two-Higgs-doublet models to the GUT scale implies essentially no constraints on the masses of light neutral and charged higgsinos, the superpartners of the two Higgs doublets. Hence, a discovery of just light higgsinos at the LHC could be interpreted as a possible hint for a supersymmetric UV completion at the grand unification scale.

The Standard Model requires fine-tuning of the cosmological constant and the Higgs mass. In two-Higgs-doublet models also the mass term of the second Higgs doublet has to be fine-tuned. This situation is unsatisfactory. It is conceivable that an explanation of this puzzle will eventually be provided by the UV completion.

\section{Acknowledgments}

This work has been supported by the German Science Foundation (DFG) within the Collaborative Research Center 676 "Particles, Strings and the Early Universe". This research was also supported in part by the European Commission through the "HiggsTools" Initial Training Network PITN-GA-2012-316704. We thank J. Bernon, S. Kraml and S. Shirai for useful discussions. FB thanks the DESY theory group for hospitality.

\section{A Details on the matching at the weak scale}

In the following the applied procedure for the matching at the weak scale is described. The matching is performed at the scale $M_{t}$. 
The $\overline{\mathrm{MS}}$ gauge couplings $g_{i}\left(M_{t}\right)$ of the THDM are calculated as

$$
\begin{aligned}
& g_{1}\left(M_{t}\right)=\sqrt{\frac{5}{3} \frac{\sqrt{4 \pi \alpha_{\mathrm{em}}^{\mathrm{THDM}}\left(M_{t}\right)}}{\cos \theta_{W}},} \\
& g_{2}\left(M_{t}\right)=\frac{\sqrt{4 \pi \alpha_{\mathrm{em}}^{\mathrm{THDM}}\left(M_{t}\right)}}{\sin \theta_{W}}, \\
& g_{3}\left(M_{t}\right)=\sqrt{4 \pi \alpha_{\mathrm{s}}^{\mathrm{THDM}}\left(M_{t}\right)},
\end{aligned}
$$

where $\alpha_{\mathrm{em}}^{\mathrm{THDM}}$ and $\alpha_{\mathrm{s}}^{\mathrm{THDM}}$ denote the electromagnetic and strong coupling constants of the THDM, respectively, and $\theta_{W}$ is the $\overline{\mathrm{MS}}$ weak mixing angle. The coupling constants of the THDM are related to the corresponding Standard Model ones, $\alpha_{\mathrm{em}}^{\mathrm{SM}(5), \overline{\mathrm{MS}}}\left(M_{t}\right)$ and $\alpha_{\mathrm{S}}^{\mathrm{SM}(5), \overline{\mathrm{MS}}}\left(M_{t}\right)$, via the relation

$$
\begin{aligned}
& \alpha_{\mathrm{em}}^{\mathrm{THDM}}\left(M_{t}\right)=\frac{\alpha_{\mathrm{em}}^{\mathrm{SM}(5), \overline{\mathrm{MS}}}\left(M_{t}\right)}{1-\Delta \alpha_{\mathrm{em}}\left(M_{t}\right)}, \\
& \alpha_{\mathrm{s}}^{\mathrm{THDM}}\left(M_{t}\right)=\frac{\alpha_{\mathrm{s}}^{\mathrm{SM}(5), \overline{\mathrm{MS}}}\left(M_{t}\right)}{1-\Delta \alpha_{\mathrm{s}}\left(M_{t}\right)},
\end{aligned}
$$

where the threshold corrections $\Delta \alpha_{i}\left(\mu_{r}\right)$ read

$$
\begin{aligned}
\Delta \alpha_{\mathrm{em}}\left(\mu_{r}\right) & =\frac{\alpha_{\mathrm{em}}}{2 \pi}\left[-\frac{16}{9} \log \frac{m_{t}}{\mu_{r}}-\frac{4}{3} \sum_{i=1}^{2} \log \frac{m_{\tilde{\chi}_{i}^{ \pm}}}{\mu_{r}}-\frac{1}{3} \log \frac{m_{H^{ \pm}}}{\mu_{r}}\right] \\
\Delta \alpha_{\mathrm{s}}\left(\mu_{r}\right) & =\frac{\alpha_{\mathrm{s}}}{2 \pi}\left[-\frac{2}{3} \log \frac{m_{t}}{\mu_{r}}-2 \log \frac{m_{\tilde{g}}}{\mu_{r}}\right] .
\end{aligned}
$$

The terms involving the masses of the charginos and the gluino are only present if these particles have not been integrated out at the high-scale and are thus part of the low-energy effective theory. As input, we use $\alpha_{\mathrm{em}}^{\mathrm{SM}(5), \overline{\mathrm{MS}}}\left(M_{Z}\right)=1 / 127.940[34]$ and $\alpha_{\mathrm{S}}^{\mathrm{SM}(5), \overline{\mathrm{MS}}}\left(M_{Z}\right)=$ 0.1184 [35], which are evolved to the scale $M_{t}$ using the 1-loop QED and 3-loop QCD $\beta$-functions in the Standard Model with 5 active quark flavours.

The $\overline{\mathrm{MS}}$ weak mixing angle $\theta_{W}$ in the THDM is determined from the Fermi constant $G_{F}=1.16638 \cdot 10^{-5}[34]$ and $M_{Z}=91.1876 \mathrm{GeV}$ [34] using the iterative approach described in [29] taking into account the full 1-loop THDM corrections and leading 2-loop Standard Model corrections to $\Delta \hat{\rho}$ and $\Delta \hat{r}[29,37]$. The vertex and box contributions, $\delta_{\mathrm{VB}}$, from potential non-Standard Model particles are neglected here.

The $\overline{\mathrm{MS}}$ Yukawa couplings $y_{i}\left(M_{t}\right)$ of the THDM are determined from the corresponding THDM $\overline{\mathrm{MS}}$ masses $m_{i}$ using the relations

$$
y_{i}\left(M_{t}\right)= \begin{cases}m_{i}\left(M_{t}\right) / v_{u}\left(M_{t}\right) & \text { if } i \text { is an up-type fermion } \\ m_{i}\left(M_{t}\right) / v_{d}\left(M_{t}\right) & \text { if } i \text { is a down-type fermion }\end{cases}
$$

The top quark $\overline{\mathrm{MS}}$ mass in the THDM is calculated from the top pole mass $M_{t}=$ 173.34 GeV [36] using the full 1-loop self-energy plus 2-loop Standard Model QCD cor- 
rections,

$$
\begin{aligned}
m_{t}\left(M_{t}\right)=M_{t}+ & \operatorname{Re} \Sigma_{t}^{S}\left(p^{2}=M_{t}^{2}, \mu_{r}=M_{t}\right) \\
+M_{t}[ & \operatorname{Re} \Sigma_{t}^{L}\left(p^{2}=M_{t}^{2}, \mu_{r}=M_{t}\right)+\operatorname{Re} \Sigma_{t}^{R}\left(p^{2}=M_{t}^{2}, \mu_{r}=M_{t}\right) \\
& \left.\quad+\Delta m_{t}^{(1), \mathrm{qcd}}\left(M_{t}\right)+\Delta m_{t}^{(2), \mathrm{qcd}}\left(M_{t}\right)\right],
\end{aligned}
$$

where $\Sigma_{t}^{S, L, R}$ denote the scalar, left- and right-handed parts of the top self-energy in the $\overline{\mathrm{MS}}$ scheme without the gluon contribution, and $\Delta m_{t}^{(1), \mathrm{qcd}}$ and $\Delta m_{t}^{(2), \text { qcd }}$ are 1- and 2-loop gluon corrections taken from ref. [30],

$$
\begin{aligned}
& \Delta m_{t}^{(1), \mathrm{qcd}}\left(\mu_{r}\right)=-\frac{g_{3}^{2}}{12 \pi^{2}}\left[4-3 \log \left(\frac{m_{t}^{2}}{\mu_{r}^{2}}\right)\right] \\
& \Delta m_{t}^{(2), \mathrm{qcd}}\left(\mu_{r}\right)=\left(\Delta m_{t}^{(1), \mathrm{qcd}}\right)^{2}-\frac{g_{3}^{4}}{4608 \pi^{4}}\left[\begin{array}{l}
396 \log ^{2} \frac{m_{t}^{2}}{\mu_{r}^{2}}-2028 \log \frac{m_{t}^{2}}{\mu_{r}^{2}}-48 \zeta(3) \\
\left.+2821+16 \pi^{2}(1+\log 4)\right]
\end{array}\right.
\end{aligned}
$$

The bottom quark $\overline{\mathrm{MS}}$ mass in the THDM, $m_{b}\left(M_{t}\right)$, is obtained from the $\overline{\mathrm{MS}}$ mass $m_{b}^{\mathrm{SM}(5)}\left(m_{b}\right)=4.18 \mathrm{GeV}$ in the Standard Model with 5 active quark flavours by first evolving $m_{b}^{\mathrm{SM}(5)}\left(m_{b}\right)$ to the scale $M_{t}$ using the 1-loop QED and 3-loop QCD RGE. Afterwards, $m_{b}^{\mathrm{SM}(5)}\left(M_{t}\right)$ is converted to $m_{b}\left(M_{t}\right)$ as

$$
\begin{aligned}
m_{b}\left(M_{t}\right)= & \frac{m_{b}^{\mathrm{SM}(5)}\left(M_{t}\right)}{1-\Delta m_{b}}, \\
\Delta m_{b}= & \operatorname{Re} \Sigma_{b}^{S}\left(p^{2}=m_{b}^{2}, \mu_{r}=M_{t}\right) / m_{b} \\
& +\operatorname{Re} \Sigma_{b}^{L}\left(p^{2}=m_{b}^{2}, \mu_{r}=M_{t}\right)+\operatorname{Re} \Sigma_{b}^{R}\left(p^{2}=m_{b}^{2}, \mu_{r}=M_{t}\right),
\end{aligned}
$$

where $\Sigma_{b}^{S, L, R}$ are the scalar, left- and right-handed parts of the 1-loop bottom quark selfenergy in the $\overline{\mathrm{MS}}$ scheme in which all Standard Model particles, except the bottom quark, the top quark and the $\mathrm{W}, \mathrm{Z}$ and Higgs bosons, are omitted. Finally, the $\overline{\mathrm{MS}}$ mass of the $\tau$ lepton in the THDM, $m_{\tau}\left(M_{t}\right)$, is calculated by first identifying the $\tau$ pole mass, $M_{\tau}$, with the $\overline{\mathrm{MS}}$ mass in the Standard Model with 5 active quark flavours at the scale $M_{\tau}$,

$$
m_{\tau}^{\mathrm{SM}(5)}\left(M_{\tau}\right)=M_{\tau}
$$

In this identification, the 1-loop Standard Model electroweak corrections to $m_{\tau}^{\mathrm{SM}(5)}\left(M_{\tau}\right)$ are neglected. Afterwards, $m_{\tau}^{\mathrm{SM}(5)}\left(M_{\tau}\right)$ is evolved to $M_{t}$ using the 1-loop QED RGE and $m_{\tau}^{\operatorname{SM}(5)}\left(M_{t}\right)$ is converted to $m_{\tau}\left(M_{t}\right)$ as

$$
\begin{aligned}
m_{\tau}\left(M_{t}\right)= & m_{\tau}^{\mathrm{SM}(5)}\left(M_{t}\right)+\operatorname{Re} \Sigma_{\tau}^{S}\left(p^{2}=m_{\tau}^{2}, \mu_{r}=M_{t}\right) \\
& +m_{\tau}^{\mathrm{SM}(5)}\left(M_{t}\right)\left[\operatorname{Re} \Sigma_{\tau}^{L}\left(p^{2}=m_{\tau}^{2}, \mu_{r}=M_{t}\right)+\operatorname{Re} \Sigma_{\tau}^{R}\left(p^{2}=m_{\tau}^{2}, \mu_{r}=M_{t}\right)\right],
\end{aligned}
$$


where $\Sigma_{\tau}^{S, L, R}$ are the scalar, left- and right-handed parts of the 1-loop $\tau$ self-energy in the $\overline{\mathrm{MS}}$ scheme where all Standard Model particles, except the $\tau$ lepton, the top quark and the W, Z and Higgs bosons, are omitted.

The $\overline{\mathrm{MS}}$ vacuum expectation values $v_{u}\left(M_{t}\right)$ and $v_{d}\left(M_{t}\right)$ are obtained from the running $\mathrm{Z}$ mass, $m_{Z}\left(M_{t}\right)$ and the $\overline{\mathrm{MS}}$ gauge couplings via

$$
\begin{aligned}
& v_{d}\left(M_{t}\right)=\frac{\sqrt{2} m_{Z}\left(M_{t}\right)}{\sqrt{3 / 5 g_{1}^{2}\left(M_{t}\right)+g_{2}^{2}\left(M_{t}\right)} \cos \beta\left(M_{t}\right)}, \\
& v_{u}\left(M_{t}\right)=\frac{\sqrt{2} m_{Z}\left(M_{t}\right)}{\sqrt{3 / 5 g_{1}^{2}\left(M_{t}\right)+g_{2}^{2}\left(M_{t}\right)} \sin \beta\left(M_{t}\right)},
\end{aligned}
$$

where the running $\mathrm{Z}$ mass is given by

$$
m_{Z}^{2}\left(M_{t}\right)=M_{Z}^{2}+\operatorname{Re} \Sigma_{Z Z}^{T}\left(p^{2}=M_{Z}^{2}, \mu_{r}=M_{t}\right),
$$

and $\Sigma_{Z Z}^{T}$ is the transverse part of the 1-loop Z self-energy in the THDM including higgsinos and gauginos if present in the theory.

As shown above, the matching at the weak scale at the 1- and 2-loop level introduces a dependency of the gauge and Yukawa couplings as well as the vacuum expectation values on the particle spectrum of the THDM (possibly including higgsinos and gauginos). These gauge and Yukawa couplings enter the renormalisation group equations for all model parameters, including the quartic couplings $\lambda_{i}$, which are fixed by boundary conditions at the high scale, $M_{S}$. For this reason, an iteration between the matching of the $\lambda_{i}$ at $M_{S}$ and the matching to the Standard Model at $M_{t}$ must be performed until a convergent solution to this boundary value problem has been found.

If a consistent solution to this boundary value problem has been found, the pole mass spectrum is calculated at the 1-loop level. This calculation follows a similar procedure as described in ref. [29] for the MSSM, adapted to the THDM case, potentially including higgsinos and gauginos, if present in the theory.

\section{B Vacuum (meta)stability}

Absolute stability of the electroweak vacuum is a strong requirement. From the phenomenological point of view, it might be more reasonable to demand metastability with a lifetime larger than the age of the universe. Semiclassically, the tunnelling probability into the true vacuum during a cosmic time $\tau$ (or more precisely, the tunnelling rate times $\tau$ ) can be estimated as [31]

$$
p \sim\left(\frac{\tau}{R}\right)^{4} e^{-S_{\text {bounce }}}
$$

where $S_{\text {bounce }}$ is the euclidean action of the "bounce" instanton solution which interpolates between the false and the true vacuum, and $R$ is the characteristic size of the bubble. Note that, at this level, $R$ is undetermined for a classically scale invariant potential.

A more precise estimate in quantum theory was discussed e.g. in ref. [32] for the case of the Standard Model. Following their analysis, for a single scalar field with a $\phi^{4}$ potential 
(neglecting the Higgs mass term),

$$
\mathcal{L}=\frac{1}{2}\left(\partial_{\mu} \phi\right)^{2}-\frac{\lambda}{4} \phi^{4},
$$

the tunnelling probability for negative $\lambda$ can be estimated as

$$
p \approx \max _{R}\left(\frac{\tau}{R}\right)^{4} \exp \left(-\frac{8 \pi^{2}}{3\left|\lambda\left(\frac{1}{R}\right)\right|}+\Delta S\right),
$$

where $\lambda\left(\mu_{r}\right)$ is the running quartic coupling, and $\Delta S$ are one-loop corrections from particles coupling to $\phi$. We require $p \ll 1$ when $\tau$ is the age of the universe, $\tau=10^{10} \mathrm{yr}$. The tunnelling probability is dominated by the largest value of $|\lambda|$, which, for the Standard Model, leads to a condition that $\lambda$ be larger than about -0.05 during its entire RG evolution up to $M_{\text {Planck }}[32]$ (somewhat larger $|\lambda|$ being permissible at low scales).

In our case the model is somewhat more complicated as it involves several scalar degrees of freedom. However, out of the four conditions for absolute stability eqs. (2.5)-(2.8), the first three turn out always to be satisfied as a consequence of the supersymmetric boundary conditions on the quartics. The remaining condition eq. (2.8)

$$
\tilde{\lambda} \equiv \lambda_{3}+\lambda_{4}+\sqrt{\lambda_{1} \lambda_{2}}>0
$$

may be violated, which corresponds to one particular direction in field space becoming unstable. To see this explicitly, we follow ref. [33] and set

$$
a=H_{1}^{\dagger} H_{1}, \quad b=H_{2}^{\dagger} H_{2}, \quad c=\operatorname{Re} H_{1}^{\dagger} H_{2}, \quad d=\operatorname{Im} H_{1}^{\dagger} H_{2} .
$$

This allows us to write the quartic potential as the sum of three terms which are manifestly positive definite if the stability conditions eqs. (2.5)-(2.8) are satisfied:

$$
V_{4}=\frac{1}{2}\left(\sqrt{\lambda_{1}} a-\sqrt{\lambda_{2}} b\right)^{2}+\left(\lambda_{3}+\sqrt{\lambda_{1} \lambda_{2}}\right)\left(a b-c^{2}-d^{2}\right)+\tilde{\lambda}\left(c^{2}+d^{2}\right) .
$$

If however $\tilde{\lambda}$ is negative, then the potential is unbounded from below along the direction $a=\sqrt{\lambda_{2} / \lambda_{1}} b, a b=c^{2}+d^{2}$ with $c^{2}+d^{2}$ growing large.

To map this onto a one-dimensional problem, we choose a gauge and a field basis such that

$$
H_{1}=\left(\begin{array}{c}
0 \\
\frac{1}{\sqrt{2}}(\phi \cos \theta+\chi \sin \theta) e^{i \xi_{1}}
\end{array}\right), \quad H_{2}=\left(\begin{array}{c}
\frac{\rho}{\sqrt{2}} e^{i \xi_{2}} \\
\frac{1}{\sqrt{2}}(-\phi \sin \theta+\chi \cos \theta) e^{i \xi_{3}}
\end{array}\right),
$$

where $\phi, \chi, \rho$, and $\xi_{i}$ are real and $\theta$ is defined by

$$
\frac{1+\sin (2 \theta)}{1-\sin (2 \theta)}=\sqrt{\frac{\lambda_{2}}{\lambda_{1}}} .
$$

Choosing $\rho=0$ and $\chi=\phi$ sets the first two terms in eq. (B.5) to zero. The remaining effective potential along the $\phi$ direction is

$$
V_{\mathrm{eff}}(\phi)=\frac{\tilde{\lambda}}{4} \cos ^{2}(2 \theta) \phi^{4},
$$


or equivalently

$$
V_{\text {eff }}(\phi)=\frac{\lambda}{4} \phi^{4}, \quad \text { where } \lambda=\frac{4 \sqrt{\lambda_{1} \lambda_{2}}\left(\lambda_{3}+\lambda_{4}+\sqrt{\lambda_{1} \lambda_{2}}\right)}{\lambda_{1}+\lambda_{2}+2 \sqrt{\lambda_{1} \lambda_{2}}} .
$$

The tunnelling rate will be dominated by bounces along this line in field space, so the problem is effectively one-dimensional. Using for $S_{\text {bounce }}$ the RG-improved one-dimensional expression without further loop corrections,

$$
S_{\text {bounce }}=\frac{8 \pi^{2}}{3\left|\lambda\left(\mu_{r}\right)\right|},
$$

we obtain a reasonably accurate necessary condition for the longevity of the electroweak vacuum from eq. (B.3). The condition is that at all scales $\mu_{r}$ between the electroweak scale and $M_{S}$ we should have the inequality

$$
\lambda\left(\mu_{r}\right) \gtrsim-\frac{2.82}{41.1+\log _{10} \frac{\mu_{r}}{\mathrm{GeV}}},
$$

with $\lambda$ defined in eq. (B.9). This lower bound on $\lambda$ varies between -0.065 at the electroweak scale and -0.047 at $\mu_{r}=M_{\text {Planck }}$. It could probably be strengthened slightly by going beyond our simple one-dimensional approximation.

Open Access. This article is distributed under the terms of the Creative Commons Attribution License (CC-BY 4.0), which permits any use, distribution and reproduction in any medium, provided the original author(s) and source are credited.

\section{References}

[1] M.B. Green, J.H. Schwarz and E. Witten, Superstring Theory. Vol. 2: Loop Amplitudes, Anomalies And Phenomenology, Cambridge University Press (1987) [InSPIRE].

[2] L.E. Ibanez and A.M. Uranga, String theory and particle physics: An introduction to string phenomenology, Cambridge University Press (2012).

[3] L.J. Hall and Y. Nomura, A Finely-Predicted Higgs Boson Mass from A Finely-Tuned Weak Scale, JHEP 03 (2010) 076 [arXiv:0910.2235] [INSPIRE].

[4] N. Arkani-Hamed and S. Dimopoulos, Supersymmetric unification without low energy supersymmetry and signatures for fine-tuning at the LHC, JHEP 06 (2005) 073 [hep-th/0405159] [INSPIRE].

[5] G.F. Giudice and A. Romanino, Split supersymmetry, Nucl. Phys. B 699 (2004) 65 [Erratum ibid. B 706 (2005) 487] [hep-ph/0406088] [INSPIRE].

[6] I. Antoniadis and S. Dimopoulos, Splitting supersymmetry in string theory, Nucl. Phys. B 715 (2005) 120 [hep-th/0411032] [INSPIRE].

[7] W. Buchmüller, M. Dierigl, F. Ruehle and J. Schweizer, Split symmetries, Phys. Lett. B 750 (2015) 615 [arXiv: 1507.06819] [INSPIRE].

[8] G.F. Giudice and A. Strumia, Probing High-Scale and Split Supersymmetry with Higgs Mass Measurements, Nucl. Phys. B 858 (2012) 63 [arXiv:1108.6077] [InSPIRE]. 
[9] E. Bagnaschi, G.F. Giudice, P. Slavich and A. Strumia, Higgs Mass and Unnatural Supersymmetry, JHEP 09 (2014) 092 [arXiv: 1407.4081] [INSPIRE].

[10] D. Buttazzo et al., Investigating the near-criticality of the Higgs boson, JHEP 12 (2013) 089 [arXiv:1307.3536] [INSPIRE].

[11] M. Gorbahn, S. Jager, U. Nierste and S. Trine, The supersymmetric Higgs sector and $B-\bar{B}$ mixing for large tan $\beta$, Phys. Rev. D 84 (2011) 034030 [arXiv:0901.2065] [InSPIRE].

[12] G. Lee and C.E.M. Wagner, Higgs bosons in heavy supersymmetry with an intermediate $m_{A}$, Phys. Rev. D 92 (2015) 075032 [arXiv: 1508.00576] [INSPIRE].

[13] N. Chakrabarty, U.K. Dey and B. Mukhopadhyaya, High-scale validity of a two-Higgs doublet scenario: a study including LHC data, JHEP 12 (2014) 166 [arXiv:1407.2145] [INSPIRE].

[14] D. Das and I. Saha, Search for a stable alignment limit in two-Higgs-doublet models, Phys. Rev. D 91 (2015) 095024 [arXiv:1503.02135] [INSPIRE].

[15] D. Chowdhury and O. Eberhardt, Global fits of the two-loop renormalized Two-Higgs-Doublet model with soft $Z_{2}$ breaking, JHEP 11 (2015) 052 [arXiv:1503.08216] [INSPIRE].

[16] P. Ferreira, H.E. Haber and E. Santos, Preserving the validity of the Two-Higgs Doublet Model up to the Planck scale, Phys. Rev. D 92 (2015) 033003 [arXiv:1505.04001] [INSPIRE].

[17] P. Athron, J.-h. Park, D. Stöckinger and A. Voigt, FlexibleSUSY - A spectrum generator generator for supersymmetric models, Comput. Phys. Commun. 190 (2015) 139 [arXiv: 1406.2319] [INSPIRE].

[18] Heavy Flavor Averaging Group (HFAG) collaboration, Y. Amhis et al., Averages of b-hadron, c-hadron and $\tau$-lepton properties as of summer 2014, arXiv:1412.7515 [INSPIRE].

[19] ATLAS collaboration, Search for neutral Higgs bosons of the minimal supersymmetric standard model in pp collisions at $\sqrt{s}=8 \mathrm{TeV}$ with the ATLAS detector, JHEP 11 (2014) 056 [arXiv: 1409.6064] [INSPIRE].

[20] CMS collaboration, Search for additional neutral Higgs bosons decaying to a pair of tau leptons in pp collisions at $\sqrt{s}=7$ and $8 \mathrm{TeV}$, CMS-PAS-HIG-14-029.

[21] ATLAS collaboration, Search for Neutral Minimal Supersymmetric Standard Model Higgs Bosons $H / A \rightarrow \tau \tau$ produced in pp collisions at $\sqrt{s}=13 \mathrm{TeV}$ with the ATLAS Detector, ATLAS-CONF-2015-061.

[22] H.E. Haber and R. Hempfling, The Renormalization group improved Higgs sector of the minimal supersymmetric model, Phys. Rev. D 48 (1993) 4280 [hep-ph/9307201] [INSPIRE].

[23] N.G. Deshpande and E. Ma, Pattern of Symmetry Breaking with Two Higgs Doublets, Phys. Rev. D 18 (1978) 2574 [inSPIRE].

[24] F. Staub, SARAH, arXiv:0806.0538 [INSPIRE].

[25] F. Staub, Automatic Calculation of supersymmetric Renormalization Group Equations and Self Energies, Comput. Phys. Commun. 182 (2011) 808 [arXiv: 1002.0840] [InSPIRE].

[26] F. Staub, SARAH 4: A tool for (not only SUSY) model builders, Comput. Phys. Commun. 185 (2014) 1773 [arXiv:1309.7223] [INSPIRE].

[27] F. Lyonnet, I. Schienbein, F. Staub and A. Wingerter, PyR@TE: Renormalization Group Equations for General Gauge Theories, Comput. Phys. Commun. 185 (2014) 1130 [arXiv:1309.7030] [INSPIRE]. 
[28] F. Lyonnet, Automation of non-SUSY two-loop RGEs with PyR@TE: latest developments, arXiv: 1510.08841 [INSPIRE].

[29] D.M. Pierce, J.A. Bagger, K.T. Matchev and R.-j. Zhang, Precision corrections in the minimal supersymmetric standard model, Nucl. Phys. B 491 (1997) 3 [hep-ph/9606211] [INSPIRE].

[30] J. Fleischer, F. Jegerlehner, O.V. Tarasov and O.L. Veretin, Two loop QCD corrections of the massive fermion propagator, Nucl. Phys. B 539 (1999) 671 [Erratum ibid. B 571 (2000) 511] [hep-ph/9803493] [INSPIRE].

[31] S.R. Coleman, The Fate of the False Vacuum. 1. Semiclassical Theory, Phys. Rev. D 15 (1977) 2929 [Erratum ibid. D 16 (1977) 1248] [INSPIRE].

[32] G. Isidori, G. Ridolfi and A. Strumia, On the metastability of the standard model vacuum, Nucl. Phys. B 609 (2001) 387 [hep-ph/0104016] [InSPIRE].

[33] J.F. Gunion and H.E. Haber, The CP conserving two Higgs doublet model: The approach to the decoupling limit, Phys. Rev. D 67 (2003) 075019 [hep-ph/0207010] [INSPIRE].

[34] Particle Data Group collaboration, K.A. Olive et al., Review of Particle Physics, Chin. Phys. C 38 (2014) 090001 [inSPIRE].

[35] S. Bethke, The 2009 World Average of $\alpha_{s}$, Eur. Phys. J. C 64 (2009) 689 [arXiv: 0908.1135] [INSPIRE].

[36] ATLAS, CDF, CMS, D0 collaborations, First combination of Tevatron and LHC measurements of the top-quark mass, arXiv:1403.4427 [INSPIRE].

[37] S. Fanchiotti, B.A. Kniehl and A. Sirlin, Incorporation of QCD effects in basic corrections of the electroweak theory, Phys. Rev. D 48 (1993) 307 [hep-ph/9212285] [INSPIRE]. 